\title{
Comparison of Proximal Contacts of Class II Resin Composite Restorations In Vitro
}

\author{
BAC Loomans • NJM Opdam • FJM Roeters \\ EM Bronkhorst • RCW Burgersdijk
}

\section{Clinical Relevance}

When placing a Class II resin composite restoration, the use of sectional matrix systems and separation rings to obtain tight proximal contacts is recommended.

\section{SUMMARY}

This study investigated the tightness of the proximal contact when placing posterior resin composite restorations with circumferential and sectional matrix systems in an in vitro model using a special measuring device (Tooth Pressure Meter). A

\footnotetext{
*BAC Loomans, DDS, assistant professor, Department of Preventive and Restorative Dentistry, Radboud University Nijmegen Medical Centre, College of Dental Sciences, Nijmegen, The Netherlands

NJM Opdam, PhD, DDS, assistant professor, Department of Preventive and Restorative Dentistry, Radboud University Nijmegen Medical Centre, College of Dental Sciences, Nijmegen, The Netherlands
}

FJM Roeters, PhD, DDS, associate professor, Department of Preventive and Restorative Dentistry, Radboud University Nijmegen Medical Centre, College of Dental Sciences, Nijmegen, The Netherlands

EM Bronkhorst, MSc, $\mathrm{PhD}$, assistant professor, Department of Preventive and Restorative Dentistry, Radboud University Nijmegen Medical Centre, College of Dental Sciences, Nijmegen, The Netherlands

RCW Burgersdijk, PhD, DDS, professor, dean, Department of Preventive and Restorative Dentistry, Radboud University Nijmegen Medical Centre, College of Dental Sciences, Nijmegen, The Netherlands

*Reprint request: PO Box 9101, 6500 HB Nijmegen, The Netherlands; e-mail: b.loomans@dent.umcn.nl

DOI: $10.2341 / 05-133$ manikin model was used with an artificial first molar in which an MO-preparation was ground, simulating the clinical situation of an amalgam replacement. This preparation was duplicated, resulting in 160 identically prepared teeth. These teeth were divided into 8 groups $(n=20)$. In 2 groups, circumferential matrix bands (flat or contoured) in a Tofflemire retainer were applied. In the remaining 6 groups, 3 different separation rings were combined with 2 types of sectional matrix bands. All the cavities were restored using Clearfil Photo Bond and Clearfil AP-X. The tightness of the proximal contact was measured using the Tooth Pressure Meter. Data were statistically analyzed using SPSS 12. ANOVA was used to find differences in proximal contact tightness between the groups. Tukey tests were used to find differences between the homogeneous subgroups. The use of sectional matrices combined with separation rings resulted in tighter proximal contacts compared to when circumferential systems were used $(p<0.001)$. The use of these devices is therefore recommended when posterior resin composite restorations are placed.

\section{INTRODUCTION}

The procedures required to place Class II resin composite or amalgam restorations are different in many aspects. With an amalgam restoration, an adequate 
proximal contact can be achieved by condensing the restorative material into the cavity and against the adjacent tooth surface. Stiff packable resin composites were introduced into the market, which claim to have some of the handling characteristics of dental amalgam. Nevertheless, due to the visco-elastic properties of resin composites, condensation of the material into the cavity is impossible. As a result, practitioners encounter difficulties reconstructing proximal contacts. However, a good proximal contact is important for a well functioning dentition. When a proximal contact is too loose, this may lead to food impaction, tooth migration, periodontal complications and carious lesions (Dörfer, 1997; Hancock \& others, 1980).

Only a few in vitro studies have investigated the role of placement techniques in creating an adequate proximal contact. A study by Klein and others (2002) has shown that the use of high viscosity composites does not improve tightness of the proximal contact over medium viscosity composites. The use of flowable composites resulted in the loosest proximal contacts, with amalgam providing the tightest proximal contacts. When an incremental technique was combined with the application of pressure, using a hand-instrument on the contact area of the matrix with the adjacent tooth during polymerization of the first layer, a tighter proximal contact was obtained compared to when a bulk-fill technique without additional pressure was applied (Dörfer, Steinhaussen \& Staehle, 1996; Eberhard, Dörfer \& Staehle, 1996; El-Badrawy \& others, 2003). The necessary interproximal separation can also be obtained by inserting wooden wedges interdentally, described as the "pre-wedging" technique (Albers, 1985). Another technique used to obtain the separation of teeth is through separation rings. In an in vitro study by Peumans and others (2001), it was found that tightness of the proximal contact of a Class II 2-surface resin composite restoration was looser with a circumferential matrix system than with a sectional matrix in combination with a separation ring. This result was confirmed in a clinical study which showed that sectional matrix systems, combined with separation rings, resulted in tighter proximal contacts, while a circumferential matrix system resulted in looser proximal contacts compared to the situation prior to treatment (Loomans \& others, 2006).

Measurement of proximal contact tightness is different for the in vivo and in vitro situation. In vivo, a large variation in proximal contact tightness appears to exist between individuals; it varies between $0.10 \mathrm{~N}$ and $12.43 \mathrm{~N}$ (Loomans \& others, 2006). Therefore, tightness of the proximal contact after application of a restorative technique can only be evaluated when the contact tightness is compared both before and after treatment. In terms of in vitro studies, a manikin model can be used to simulate clinical situations. Moreover, when restorations are made in identically shaped teeth in such a model, standardization of conditions is possible, allowing for the comparison of contact tightness of restorations after placement.

To quantify proximal contact tightness, a few methods are available. In a study by Peumans and others (2001), standardized metal blades were inserted interdentally, and the size of the thickest blade, which passed the contact area, was recorded. Another method is to use a special device (Tooth Pressure Meter) originally designed by Dörfer and others (2000). This device measures the force needed to remove a $0.05-\mathrm{mm}$ metal strip from the proximal contact, and it has been used in some in vivo studies (Dörfer \& others, 2000, 2001; Loomans \& others, 2006).

The aim of this study was to investigate the proximal contact tightness of posterior resin composite restorations placed with circumferential and sectional matrix systems in an in vitro model using the Tooth Pressure Meter. The hypothesis $\left(\mathrm{H}_{0}\right)$ tested was that the obtained proximal contact tightness of all used matrix systems was equal.

\section{METHODS AND MATERIALS}

In order to standardize the restorative procedure and simulate clinical situations, a manikin model ( $\mathrm{KaVo}$, Dental, Biberach, Germany) was used. For the experiments, the contact site between the second premolar and first molar in the lower jaw was selected. In the artificial first molar (KaVo), an MO-preparation was ground with diamond burs in a high speed handpiece. In order to simulate a clinically representative situation that replaced a moderate sized amalgam restoration, the extensions of the proximal box were $5.0 \mathrm{~mm}$ in the bucco-lingual, $6.0 \mathrm{~mm}$ in the occlusal-gingival and 1.3 $\mathrm{mm}$ in the mesial-distal directions. The occlusal step was $4.5 \mathrm{~mm}$ in buccal-lingual width, 2.5-mm deep and $6.0 \mathrm{~mm}$ in mesial-distal width. This preparation was considered a master model. Using a copy-milling machine (Celay, Mikrona Technologie AG, Spreitenbach, Switzerland), this model was then duplicated, resulting in 160 identical preparations in artificial first molars $(\mathrm{KaVo})$. The prepared teeth were placed on the manikin model (KaVo) and apically equipped with a stem-like anchoring system that allowed some mobility of the tooth to simulate normal physiological tooth mobility. In order to prevent wear of the distal surface of tooth \#35 during the restorative procedures and proximal contact tightness measurements, this tooth was duplicated by casting it in a wear-resistant chromium-cobalt alloy. In Figure 1, the in vitro set-up is shown.

Table 1 summarizes the product profiles and batch numbers of the materials used in this study, and Table 2 shows the 8 different groups in the study. In all groups consisting of 20 teeth, the matrices were secured inter- 


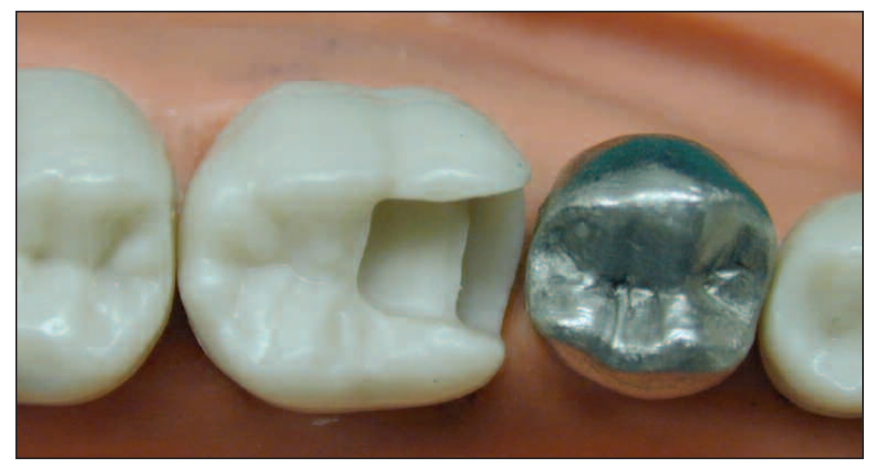

Figure 1. MO-preparation in artificial molar 36 placed in the manikin model. A metal-cast of tooth 35 prevents interproximal damage during the restorative and measuring procedure. dentally with wooden wedges (KerrHawe), and before application of the adhesive procedure, the contact area was burnished with a hand instrument (PFI 49, Weybridge, UK) so that no visual space was left between the matrix and adjacent tooth. All cavities were restored by a single operator, using an adhesive and hybrid composite (Clearfil Photo Bond and Clearfil AP-X; Kuraray Medical, Tokyo, Japan).

The adhesive system was mixed, placed on the tooth surface and cured for 10 seconds with a halogen polymerization unit (PolyLux II, KaVo, light intensity 600 $\mathrm{mW} / \mathrm{cm}^{2}$ ). Subsequently, the resin composite was injected from the compule into the cavity in 2 horizontal increments, and each layer was cured for 20 seconds from the occlusal surface. After removing the matrix, the restorations were post-cured for 20 seconds from the buccal and the lingual sides. The restorations were not finished or adjusted in order to prevent changes in the proximal surface.

To measure proximal contact tightness, the manikin model was mounted in a special device (Testor, Otto W o l p e r t - W e r k e, L u d wig s h a f e n, Germany) that allowed for a standardized measurement of all proximal contact areas as shown in Figures 2-4. Proximal contact tightness was measured using the Tooth

\begin{tabular}{|c|c|c|c|}
\hline & Matrix System & Materials & Separation Obtained By \\
\hline Group 1 & Tofflemire + circumferential flat matrix & No.1 X-thin matrix (Produits Dentaire) & Hand-instrument (PFI 49) \\
\hline Group 2 & $\begin{array}{l}\text { Tofflemire + circumferential pre-contoured } \\
\text { matrix }\end{array}$ & 1001-c matrix (Hawe Neos) & Hand-instrument (PFI 49) \\
\hline Group 3 & Flexible sectional matrix & Stiff Flex matrix (Danville Materials) & $\begin{array}{l}\text { Separation ring (Composi-Tight } \\
\text { Gold } A U 400, \text { GDS) }\end{array}$ \\
\hline Group 4 & Dead-soft sectional matrix & Lite Flex matrix (Dentsply) & $\begin{array}{l}\text { Separation ring (Composi-Tight } \\
\text { Gold } A U 400, \text { GDS) }\end{array}$ \\
\hline Group 5 & Flexible sectional matrix & Stiff Flex matrix (Danville Materials) & $\begin{array}{l}\text { Separation ring (Contact Matrix } \\
\text { System, Danville Materials) }\end{array}$ \\
\hline Group 6 & Dead-soft sectional matrix & Lite Flex matrix (Dentsply) & $\begin{array}{l}\text { Separation ring (Contact Matrix } \\
\text { System, Danville Materials) }\end{array}$ \\
\hline Group 7 & Flexible sectional matrix & Stiff Flex matrix (Danville Materials) & $\begin{array}{l}\text { Separation ring (Palodent BiTine } \\
\text { type 1, Dentsply) }\end{array}$ \\
\hline Group 8 & Dead-soft sectional matrix & Lite Flex matrix (Dentsply) & $\begin{array}{l}\text { Separation ring (Palodent BiTine } \\
\text { type 1, Dentsply) }\end{array}$ \\
\hline
\end{tabular}



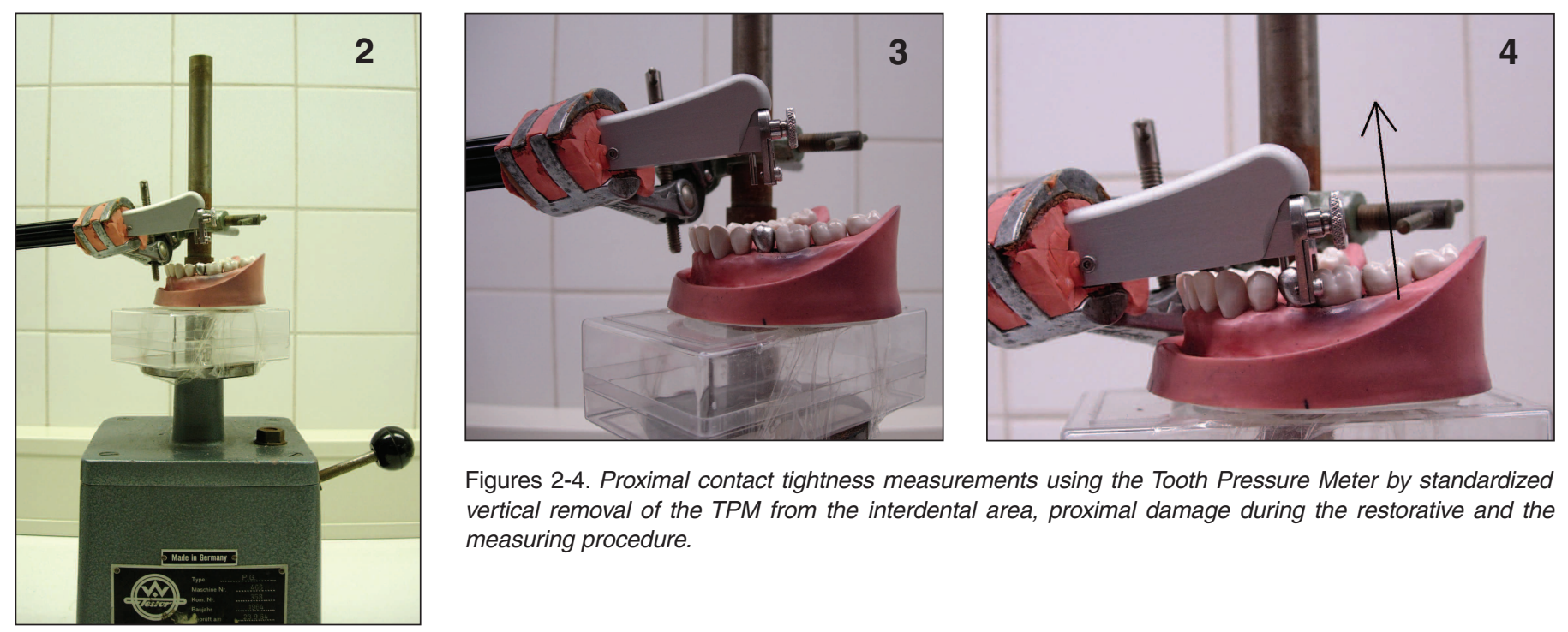

Figures 2-4. Proximal contact tightness measurements using the Tooth Pressure Meter by standardized vertical removal of the TPM from the interdental area, proximal damage during the restorative and the measuring procedure.

\begin{tabular}{|c|c|c|c|c|c|}
\hline & Circumferential Matrix Systems & $\mathbf{n}$ & Tightness [N] & SEM [N] & $95 \% \mathrm{Cl}[\mathrm{N}]$ \\
\hline Group 1 & Flat \#1 X-thin matrix with hand-instrument & 20 & $2.89^{\mathrm{a}}$ & 0.25 & {$[2.38 \ldots 3.41]$} \\
\hline \multirow[t]{2}{*}{ Group 2} & $\begin{array}{l}\text { Pre-contoured 1001-c matrix with hand- } \\
\text { instrument }\end{array}$ & 20 & $3.42^{\mathrm{a}}$ & 0.30 & {$[2.79 \ldots 4.04]$} \\
\hline & Sectional Matrix Systems & & & & \\
\hline Group 3 & $\begin{array}{l}\text { Composi-Tight Gold + Flexible sectional } \\
\text { matrix }\end{array}$ & 20 & $8.86^{d}$ & 0.45 & {$[7.91 \ldots 9.81]$} \\
\hline Group 4 & $\begin{array}{l}\text { Composi-Tight Gold + Dead-soft sectional } \\
\text { matrix }\end{array}$ & 20 & $7.13^{\mathrm{bc}}$ & 0.36 & {$[6.38 \ldots 7.88]$} \\
\hline Group 5 & $\begin{array}{l}\text { Contact Matrix System + Flexible sectional } \\
\text { matrix }\end{array}$ & 20 & $6.60^{\mathrm{bc}}$ & 0.15 & {$[6.29 \ldots 6.92]$} \\
\hline Group 6 & $\begin{array}{l}\text { Contact Matrix System + Dead-soft sectional } \\
\text { matrix }\end{array}$ & 20 & $6.07^{\mathrm{b}}$ & 0.58 & {$[4.86 \ldots 7.28]$} \\
\hline Group 7 & Palodent BiTine + Flexible sectional matrix & 20 & $8.02^{\text {cd }}$ & 0.31 & {$[7.37 \ldots 8.67]$} \\
\hline Group 8 & Palodent BiTine + Dead-soft sectional matrix & 20 & $5.67^{\mathrm{b}}$ & 0.56 & {$[4.49 \ldots 6.84]$} \\
\hline
\end{tabular}

\begin{tabular}{|c|c|c|c|c|}
\hline & & & & \\
\hline Effect of Ring Independent of Used Sectional Matrix & $\mathbf{n}$ & Tightness $[\mathbf{N}]$ & SEM $[\mathbf{N}]$ & $95 \% \mathrm{Cl}[\mathrm{N}]$ \\
\hline Contact Matrix System (Danville Materials) & 40 & $6.34^{a}$ & 0.30 & {$[5.73 \ldots 6.94]$} \\
\hline Palodent BiTine (Dentsply) & 40 & $6.84^{\mathrm{a}}$ & 0.37 & {$[6.10 \ldots .7 .59]$} \\
\hline Composi-Tight Gold (GDS) & 40 & $7.99^{b}$ & 0.32 & {$[7.35 \ldots 8.64]$} \\
\hline \multicolumn{5}{|l|}{ Effect of Matrix Independent of Used Ring } \\
\hline Dead-soft matrix & 60 & $6.29^{\circ}$ & 0.30 & {$[5.69 \ldots 6.89]$} \\
\hline Flexible matrix & 60 & $7.83^{\mathrm{d}}$ & 0.22 & {$[7.38 \ldots 8.27]$} \\
\hline
\end{tabular}

Pressure Meter. This device uses a 0.05-mm thick metal strip that is inserted interdentally from the occlusal direction. The tightness of the proximal contact is quantified as the maximum frictional force $[\mathrm{N}]$ when the strip is slowly removed in the occlusal (vertical) direction. The final result for a measuring site was the mean value of 5 consecutive measuring procedures. Each procedure included removal and repositioning of the tooth 
in the manikin model, followed by 3 consecutive contact tightness measurements with the Tooth Pressure Meter. Sometimes a measurement failed, for example, due to deformations in the strip or non-parallel removal of the strip from the interdental area, which resulted in an outcome exceeding the maximum (pre-set) range among the 3 measurements of $0.5 \mathrm{~N}$. This measurement was then excluded from the analysis, and this led to a repetition of the measurement. Custom-written software in Excel (MS Office 2000, Windows) was used for data acquisition and to construct diagrams that related force (N) to seconds.

Data were statistically analyzed using SPSS 12 . To find differences in proximal contact tightness between groups, ANOVA was used. If the $p$-value was $\leq 5 \%$, Tukey tests were used to form sets of homogeneous subgroups within the groups tested.

\section{RESULTS}

In Table 3, the mean proximal contact tightness of all groups is shown. The use of sectional matrices combined with separation rings in Groups 3-8 resulted in statistically significantly tighter proximal contacts than when circumferential systems were used in combination with pressure of a hand-instrument in Groups 1 and 2 (for all comparisons: $p<0.001$ ). No statistically significant differences were found in contact tightness between the pre-contoured and flat circumferential matrices in Groups 1 and $2(p=0.983)$. To analyze the effects of the different material characteristics of the sectional matrices, the obtained proximal contact tightness of the flexible and dead-soft matrices were compared independent of the applied separation ring as seen in Table 4 . The flexible sectional matrices produced statistically significantly tighter proximal contacts $(7.83 \pm 0.22 \mathrm{~N})$ compared to dead-soft matrices $(6.29 \pm 0.30 \mathrm{~N})(p<0.001)$. For groups where a separation ring was applied, the effects of the rings, independent of the selected sectional matrix, were compared (Table 4). The Composi-Tight Gold separation ring resulted in statistically significantly tighter mean proximal contacts $(7.99 \pm 0.32 \mathrm{~N})$ than the Contact Matrix ring $(6.34 \pm 0.30 \mathrm{~N})$ or Palodent BiTine ring $(6.84 \pm 0.37 \mathrm{~N})(p=0.039$ resp $p=0.002)$. No statistically significant difference was found between the Contact Matrix ring and the Palodent BiTine ring ( $p=0.525)$.

\section{DISCUSSION}

In this study, different matrix systems and techniques were used to create proximal contacts for posterior resin composite restorations. The hypothesis tested was that the tightness of obtained proximal contacts was equal for all groups. As statistically significant differences were found, this hypothesis $\left(\mathrm{H}_{0}\right)$ had to be rejected.

In this study, a new in vitro model was designed to measure proximal contact tightness of Class II resin composite restorations. In order to establish validity of this model, the same techniques, as applied in a randomized clinical trial by Loomans and others (2006), were used in Groups 2, 5 and 8. As mentioned in the introduction, in the clinical trial, a quantitative analysis of proximal contact tightness demanded measurement of the differences between proximal contact tightness before and after treatment. In the in vitro model presented in this study, only contact tightness after treatment could be measured, as a strict standardized study set-up was followed. In both the in vivo and in vitro studies, it was found that there were statistically significant differences between the circumferential system and the 2 sectional matrix systems. Furthermore, in both studies, no statistically significant differences were found in proximal contact tightness between both sectional matrix systems. Therefore, the authors conclude that the results of both studies strongly suggest that the in vitro model of this study is representative of the clinical situation.

The use of circumferential matrices resulted in statistically significantly looser proximal contacts as compared to the use of sectional matrices with separation rings. This might be explained by the thickness of the matrix when placing a 2-surface restoration. The total proximal thickness of the matrix, when using a circumferential system (mesial and distal), is 0.07 or $0.10 \mathrm{~mm}$, while the thickness of the sectional matrix systems is $0.04 \mathrm{~mm}$. The thickness of the matrix has to be compensated for by pressure of a hand-instrument during polymerization of the resin composite. In the case of an MOD-preparation, differences between circumferential and 2 sectional matrices will disappear. To overcome the problem of this extra thickness of the matrix when using a sectional matrix system, it would be possible to restore the mesial and distal side of the preparation separately from one another. Another explanation for the differences found in proximal contact tightness between circumferential and sectional matrices is application of the separation ring. Traditionally, it is recommended to apply wedges even before starting the preparation (Albers, 1985). However, in the clinical trial and in the in vitro study, the separating effect of the wedge could not be proven; whereas, the groups using separation rings produced the tightest proximal contacts (Loomans \& others, 2006). This implies that it is probably advantageous to also combine separation rings with the circumferential matrix systems. This will be investigated in a future study.

Statistically significantly tighter proximal contacts were found when flexible matrices were used instead of dead-soft matrices. Both matrices consist of stainless steel, but the flexible matrix is resilient and stiffer, while the dead-soft matrix deforms easily. After insertion of the matrix and placement of the separation ring, the flexible matrix may better preserve the pre-con- 
toured proximal form, resulting in a tighter proximal contact. When the dead-soft matrix was used, sometimes a negative-contour of the proximal area was observed due to matrix deformation.

Clinically, it was found that sectional matrices, in combination with the Palodent BiTine or Contact Matrix System separation rings, resulted in an increase in the mean proximal contact tightness compared to the situation before treatment (Loomans \& others, 2006). In this study, it was found that, with use of the Composi-Tight Gold ring, the proximal contacts were statistically significantly tighter than for the other 2 rings, while no significant differences were found between the Palodent BiTine ring and the Contact Matrix System. These results are in accordance with clinical results (Loomans \& others, 2006). Therefore, in a clinical situation, the Composi-Tight Gold ring might result in an even tighter proximal contact compared to the other 2 rings. However, which proximal contact tightness is the most favorable is still unknown. In an ongoing clinical study, the clinical implications of alterations in contact tightness over time after treatment are monitored. Based on this study, all separation rings are able to create tight proximal contact and may help dentists solve the problem of creating an adequate proximal contact.

\section{CONCLUSIONS}

Based on the results of this in vitro study:

1. When placing Class II resin composite restorations, the use of sectional matrix systems and separation rings result in tighter proximal contacts than when traditional circumferential matrix systems are applied.

2. This new in vitro model, which uses the Tooth Pressure Meter to simulate clinical conditions when restoring Class II resin composite restorations, seems to produce reliable, clinically representative results.

\section{Acknowledgements}

The authors thank dental students Hilde Broeders and Sjoukje Wijsman for their contribution to this research.
(Received 25 September 2005)

\section{References}

Albers HF (1985) Posterior resins: Class II preparations. In: Tooth Colored Restoratives California, Cotati, Alto Books $7^{\text {th }}$ edition.

Dörfer CE, Steinhausen J \& Staehle HJ (1996) [Messung approximaler Kontaktstärken von Komposit- und Amalgamfüllungen im Seitenzahnbereich in vitro] (in German; English abstract available) Deutsches Zahnärtzliches Zeitschrift 51(6) 335-338.

Dörfer CE (1997) [Der approximal raum] (in German; English abstract available) Deutsches Zahnärtzliches Zeitschrift 52(3) 151-167.

Dörfer CE, von Bethlenfalvy ER, Staehle HJ \& Pioch T (2000) Factors influencing proximal dental contact strengths European Journal of Oral Sciences 108(5) 368-377.

Dörfer CE, Schriever A, Heidemann D, Stahle HJ \& Pioch T (2001) Influence of rubber dam on the reconstruction of proximal contacts with adhesive tooth-colored restorations Journal of Adhesive Dentistry 3(2) 169-175.

Eberhard J, Dörfer CE \& Staehle HJ (1996) [Einfluss der Inserttechnik auf approximale Kontaktstärken bei Kopositrestaurationen in vitro] (in German; English abstract available) Deutsches Zahnärtzliches Zeitschrift 51(3) 184-186.

El-Badrawy WA, Leung BW, El-Mowafy O, Rubo JH \& Rubo MH (2003) Evaluation of proximal contacts of posterior composite restorations with 4 placement techniques Journal of the Canadian Dental Association 69(3) 162-167.

Hancock EB, Mayo CV, Schwab RR \& Wirthlin MR (1980) Influence of interdental contacts on periodontal status Journal of Periodontology 51(8) 445-449.

Klein F, Keller AK, Staehle HJ \& Dörfer CE (2002) Proximal contact formation with different restorative materials and techniques American Journal of Dentistry 15(4) 232-235.

Loomans BA, Opdam NJ, Roeters FJ, Bronkhorst EM, Burgersdijk RC \& Dörfer CE (2006) A randomized clinical trial on proximal contacts of posterior composites Journal of Dentistry 34(4) 292-297.

Peumans M, Van Meerbeek B, Asscherickx K, Simon S, Abe Y, Lambrechts P \& Vanherle G (2001) Do condensable composites help to achieve better proximal contacts? Dental Materials 17(6) $533-541$. 